\title{
Design Development and Manufacture of Novel Dyeing/Washing Lab Model Machine
}

\author{
Sampath VR*, Yirdawu Zeleke, Muktarseid \\ Textile engineering department, Bahirdar University, Ethiopia
}

Submission: October 11, 2019; Published: November 01, 2019

*Corresponding author: Sampath VR, Textile Engineering department, Eitex, Salem campus, Bahirdar University, Bahirdar, Ethiopia

\begin{abstract}
Wet processing industries and laboratories were facing dyeing problems because of its unmanageable dye accessories and inadequate technology. Basically, there are a lot of laboratory materials or equipment's in a laboratory such as beakers, stoves, thermometer, and stirrers which required to be maintained. In the laboratory as a common practice both the dyeing process and the washing process are done separately at different places. This process takes space, cost and time as well. The cost of equipment's like stove is expensive and moreover very difficult to repair with inadequate supply of spares. Washing is done manually and due to the chemicals used during the dyeing process that causes hazardous to the experimenter, resulting in different skin diseases, breathing problem and so on. The other problem is the amount of MLR is limited, maximum up to $20 \mathrm{gm}$ of fabric so it is difficult or might cause uncertainty how the dye reacts on and its evenness or difficult to see if shade variation occurs on that particularly limited small sample. In this study we tried to solve two issues namely namely high cost due to material such as beaker, thermometer and expensive stoves. The Space minimization and improvement of experimenter safety is the other objective of this project and the design and development of integrated dyeing and washing machine process. Manual washing system was eliminated in the new design and methods to increase the MLR of the fabric from to $20 \mathrm{gr}$ to $1 \mathrm{~kg}$. The fabrication was conducted at EiTex laboratory, Bahirdar University, Bahirdar. The trails on the effective working of the machine were studied. The designed lab model is robust, easy to operate, safety to experimenter and at affordable cost.
\end{abstract}

Keywords: Dyeing process; Design analysis; MLR ratio; Safety; Cost analysis

\section{Introduction}

\section{Dyeing Processes}

Most textile dyeing processes initially involve transfer of the colored chemical, from the aqueous solution onto the fiber surface. It can be applied in many stages such as

a. Mass-coloration of the molten fibers. This method is for dyeing the molten fibers or plastic chips or textile polymers with pigment dyes. After that, the molten or polymers will extrude from a spinneret to form fibers. Normally, the synthetic fibers are added with white pigment in order to give a hiding power (non-see through fabrics).

b. Fiber Dyeing is the method of dyeing fibers before blending with other colors to give fancy yarns or fabrics. This is used for special purposes only.

c. Yarn Dyeing is the method of dyeing yarns in forms of hanks or packages dyeing. Coloration processes produce the most visible results of all the finishing operations carried out during the preparation of textile goods. As such they reveal both the care taken with the coloration and the degree of control exercised during other stages of the manufacturing process [1]. Fabric Dyeing is the method after weaving, knitting, or non-woven to make fabrics. This is very popular method of dyeing as the dyed fabrics will be processed further to garment industry.

Dyeing Machine is the device that is used by different industries for imparting colors. Dyeing machines come in all shapes and sizes to accommodate the various forms and quantities of textile materials. Therefore, the most important types of machine, and their problems, will be at the forefront. Dye house machinery represents a large capital investment. The correct choice of equipment, and its effective use and maintenance, are important factors influencing the success of a dye house. Modern machines often have programmable microprocessors for automatic control of the process variables, particularly the dyeing temperature [2]. According to the need of each type of substrate, different set of machines are used. The applications of this Dyeing Machine give an impetus to the products related to dyeing. However, rising en- 
ergy prices is having a knock-on effect on manufacturing expenditure for dyeing machinery. Therefore, there will be greater interest globally in machinery that has a lower energy consumption along with heat recovery systems that is able to preheat the incoming cold feed water. As the needs of customers are changing, for satisfying those different types of dyeing machine are manufactured [3].

\section{Statement of the problem}

Basically, there are a lot of laboratory materials or equipment's to be maintained in the laboratory. Materials such as beakers, stoves, thermometer, and stirrers so on. In the laboratory the experiment done most of the time involves the dyeing process and the washing process during removing the residual chemicals done separately at different place [4]. This process takes space, cost and time as well. The stove takes a space and it is expensive to buy, very difficult to repair it or even it might be very difficult to find its spares in the market even if we do it might be expensive to buy. Washing is done by manually as a result the chemicals used during the dyeing process will be hazardous to the experimenter by causing different diseases for instant skin diseases, breathing problem and so on specially if the experimenter work on a daily basis or longer hour it might cause a problem on his/ her health. The other problem is the amount of MLR is limited, maximum up to $20 \mathrm{gm}$ of fabric so it is difficult or might cause uncertainty how the dye reacts on and its evenness.

\section{General Considerations in Machine Design}

\section{Type of load and stresses caused by the load}

The load, on a machine component, may act in several ways due to which the internal stresses are set up.

\section{Motion of the parts or kinematics of the machine}

The successful operation of any machine depends largely upon the simplest arrangement of the parts which will give the motion required.

The motion of the parts may be:

a. Rectilinear motion which includes unidirectional and reciprocating motions.

b. Curvilinear motion which includes rotary, oscillatory and simple harmonic.

c. Constant velocity.

\section{Selection of materials}

It is essential that a designer should have a thorough knowledge of the properties of the materials and their behavior under working conditions. Some of the important characteristics of materials are : strength, durability, flexibility, weight, resistance to heat and corrosion, ability to cast, welded or hardened, machine ability, electrical conductivity, etc.

\section{Form and size of the parts}

The form and size are based on judgment. The smallest practicable cross-section may be used, but it may be checked that the stresses induced in the designed cross-section are reasonably safe [5]. In order to design any machine part for form and size, it is necessary to know the forces which the part must sustain. It is also important to anticipate any suddenly applied or impact load which may cause failure.

\section{Frictional Resistance and Lubrication}

There is always a loss of power due to frictional resistance and it should be noted that the friction of starting is higher than that of running friction. It is, therefore, essential that a careful attention must be given to the matter of lubrication of all surfaces which move in contact with others, whether in rotating, sliding, or rolling bearings [6].

\section{Convenient and Economical features}

In designing, the operating features of the machine should be carefully studied. The starting, controlling and stopping levers should be located based on convenient handling [7]. The adjustment for wear must be provided employing the various take up devices and arranging them so that the alignment of parts is preserved [8]. If parts are to be changed for different products or replaced on account of wear or breakage, easy access should be provided and the necessity of removing other parts to accomplish this should be avoided if possible. The economical operation of a machine which is to be used for production or for the processing of material should be studied, in order to learn whether it has the maximum capacity consistent with the production of good work [9].

\section{Use of standard parts}

The use of standard parts is closely related to cost, because the cost of standard or stock parts is only a fraction of the cost of similar parts made to order. The standard or stock parts should be used whenever possible; parts for which patterns are already in existence such as gears, pulleys and bearings and parts which may be selected from regular shop stock such as screws, nuts and pins. Bolts and studs should be as few as possible to avoid the delay caused by changing drills, reamers and taps and to decrease the number of wrenches required [10].

\section{Safety of operation}

Some machines are dangerous to operate, especially those which are speeded up to insure production at a maximum rate. Therefore, any moving part of a machine which is within the zone of a worker is considered an accident hazard and may be the cause of an injury [11]. It is, therefore, necessary that a designer should always provide safety devices for the safety of the operator. The safety appliances should in no way interfere with operation of the machine [12]. 


\section{Workshop facilities}

A design engineer should be familiar with the limitations of his employer's workshop, in order to avoid the necessity of having work done in some other workshop.

Number of machines to be manufactured: The number of articles or machines to be manufactured affects the design in a number of ways.

\section{Methodology}

\section{Data Collection and Analysis}

Table 1: Data collection and Analysis.

\begin{tabular}{|c|c|c|}
\hline Material requirements & Quantity & Specification \\
\hline Motor & 1 piece & $0.5 \mathrm{hp}$ \\
\hline Metal sheet (galvanized) & Meter & length $1 * 2 \mathrm{~m}$ with thickness of $1 \mathrm{~mm}$ and $1.25 \mathrm{~mm}$ \\
\hline Faucet & 2 pieces & $1 / 2$ \\
\hline Hallow shaft & 1 piece & Length $1 \mathrm{~m}$ \{diameter - internal $=25 \&$ External $=30 \mathrm{~mm}\}$ \\
\hline On/off switch & 1 piece & \\
\hline Clay stove with coil & 1 piece & $\mathrm{d}=60 \mathrm{~cm}$ \\
\hline Timer & 1 piece & \\
\hline Paint & 3 pieces & \\
\hline Water pipe & 2 pieces & $\mathrm{d}=25 \mathrm{~mm}$ \\
\hline Bearing & 1 piece & ball bearing model A625 \\
\hline Stirrer (fiber glass) & 1 piece & \\
\hline
\end{tabular}

This means by observing the drawbacks in textile laboratory during dyeing process such as both cost of material and space wasted is major problems. In current laboratory condition the experiment has done most of the time superlatively, the dyeing process and the washing process during removing the residual chem-

icals done separately at different place or space. Such condition is undesirable in any case since the material cost is higher and space is wasted. Non uniform, poor dyeing effect this means shade variation or unevenness occurs. So that the basic design parameters were selected and collected as follow [Table 1].

\section{Cost of construction}

The cost of construction of an article is the most important consideration involved in design.

\section{Assembling}

Every machine or structure must be assembled as a unit before it can function.

\section{Design Analysis}

\section{Mechanism or Geometry}

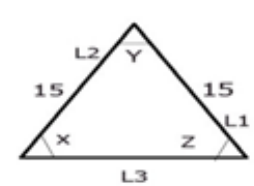

Figure 1: Stirrer angle.

Stirrer: Stirrer require in order mixing the fabric with the dye bath. It is desirable to have a larger angle in order to increase the stirring action due to centrifugal force between the dye bath and stirrer [Figure 1]. Based on our design of the stirrer we can calculate the angle and length of the stirrer as follows

$$
\begin{aligned}
& \mathrm{L}^{2}+\mathrm{L}^{2}=\mathrm{L}^{2} \ldots \ldots \ldots \ldots \ldots \ldots \ldots \ldots \ldots . . \mathrm{e}(6.1) \\
& \mathrm{L} 3=\sqrt{ } 450=21.213 \mathrm{~cm}
\end{aligned}
$$


$45+45+y=180^{\circ}$

$\mathrm{Y}=90^{\circ}$
- There for the angle of the stirrer should not be less than $45^{\circ}$ for better stirring and uniform shading of the fabric.

The length of the stirrer blade calculation [Figure 2]

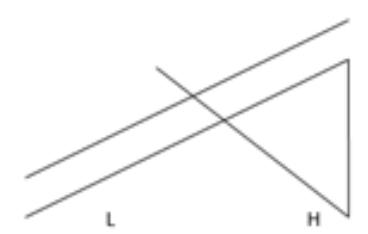

$4^{+}$

21.213

Figure 2: Stirrer Length.

Where $\mathrm{L}$ and $\mathrm{H}$ length and height of the stirrer respectively.

Tan $y=H / 21.213$

$\mathrm{H}=21.213 \tan 45^{\circ}$

$\mathrm{H}=21.213$

$\mathrm{L}^{2}=\mathrm{H}^{2}+21.213^{2}$

$\mathrm{L}=29.999 \approx 30 \mathrm{~cm}$ the total lengths of stirrer

\section{Shaft}

Shaft is rotating member usually of circular cross section which is used to transmit power and rotation motion and used to rotate stirrer.

\section{Force analysis}

Calculating force of on the shaft

$\mathrm{F}=\mathrm{mg}$....... .q (6.3)

Where $\mathrm{m}=$ mass of the motor and top cover

$\mathrm{g}=$ gravity force $(9.8 \mathrm{~m} / \mathrm{s})$

Let mass of the motor be $2 \mathrm{~kg} \mathrm{so}$,

$\mathrm{F}=2 \mathrm{~g} * 9.8 \mathrm{~m} / \mathrm{s}=19.6 \approx 20 \mathrm{~N}$.

\section{Material selection and its Specification}

The following considerations are important in material selection [13]

a. Elastic properties: stiffness and rigidity.

b. Plastic properties: yield conditions, stress-strain relations, and hysteresis.

c. Time-dependent properties: elastic phenomenon (damping capacity),creep, relaxation, and strain-rate effect. d. Fracture phenomena: crack propagation, fatigue, and ductile-to-brittle transition.

e. Thermal properties: thermal expansion, thermal conductivity, and specific heat.

f. Chemical interactions with environment: oxidation, corrosion, and diffusion.

\section{Selected Materials}

Motor: A motor is nothing but an electro-mechanical device that converts electrical energy to mechanical energy. In our case the motor use in order to transmit power is $0.5 \mathrm{Hp}$ or $0.26 \mathrm{Kw}$ power.

Shaft: A shaft is a rotating member usually of circular cross-section (solid or hollow), which is used to transmit power and rotational motion. Types of shaft use for our manufacturing is hallow shaft its inner diameter is $25 \mathrm{~mm}$ and its outer diameter is $30 \mathrm{~mm}$.

Metal sheet: Lower carbon steels are softer and more easily formed, and steels with higher carbon content are harder and stronger, but less ductile, and they become more difficult to machine and weld.

$\checkmark \quad$ Low Carbon Steel - Composition of 0.05\%-0.25\% carbon and up to $0.4 \%$ manganese. Also known as mild steel, it is a low-cost material that is easy to shape.

$\checkmark \quad$ While not as hard as higher - carbon steels, carburizing can increase its surface hardness. So that these carbons steel the most appropriate four our project.

$\checkmark \quad$ Medium Carbon Steel - Composition of 0.29\%-0.54\% carbon, with $0.60 \%-1.65 \%$ manganese. Medium carbon steel is ductile and strong, with long-wearing properties. 
$\checkmark \quad$ High Carbon Steel - Composition of 0.55\%-0.95\% carbon, with $0.30 \%-0.90 \%$ manganese. It is very strong and holds shape memory well, making it ideal for springs and wire.

$\checkmark \quad$ Very High Carbon Steel -Composition of 0.96\%-2.1\% carbon. Its high carbon content makes it an extremely strong material. Due to its brittleness, this grade requires special handling.

Bearing: Ball bearings are the most common and most used type of bearing found in a number of objects. Ball bearings are often used in individual cages to reduce friction in axle assemblies or in a series to absorb the weight placed on a moving part. A type of bearing use for our work is ball bearing model A625. The material selected for the machine is Low carbon steel since it is more cheap an easy to shape and more available in the market. Also, since we use ductile material rather than brittle materials it has a yield strength from the standard table the selected material yield strength value $=68$ psi (pound per square inch). It is defined, in general, as the ratio of the maximum stress to the working stress.

$$
\text { Factor of safety }=\frac{\text { Maximum stress }}{\text { Working or Design stress }}
$$

In case of ductile materials e.g. mild steel, where the yield point is clearly defined, the factor of safety is based upon the yield point stress.

$$
\text { Factor of safety }=\frac{\text { Yield stress }}{\text { Working or Design stress }}
$$

\section{Selection of Factor of Safety}

The selection of a proper factor of safety to be used in designing any machine component depends upon a number of considerations, such as the material, mode of manufacture, type of stress, general service conditions and shape of the parts [13] [Table 2].

Table 2: Some Values of factor of safety [13].

\begin{tabular}{|c|c|c|c|}
\hline Material & Steady load & Live load & $8-12$ \\
\hline Cast iron & $5-6$ & 7 & $16-20$ \\
\hline Wrought iron & 4 & $10-15$ \\
\hline Steel & 4 & $12-16$ \\
\hline
\end{tabular}

Based on the table value our machine material factor of safety is $=8$ since the machine is live load.

$$
\text { Factor of safety }=\frac{\text { Yield stress }}{\text { Working or Design stress }} \quad \text { from this } \quad \text { Working or Design stress }=\frac{\text { Yield stress }}{\text { Factor of safety }}=\frac{68}{8}=8.5 \text { Psi } \cdots \cdots \ldots . . . \text { e.q }(7.1)
$$

\section{Machine Design (Figure 3)}

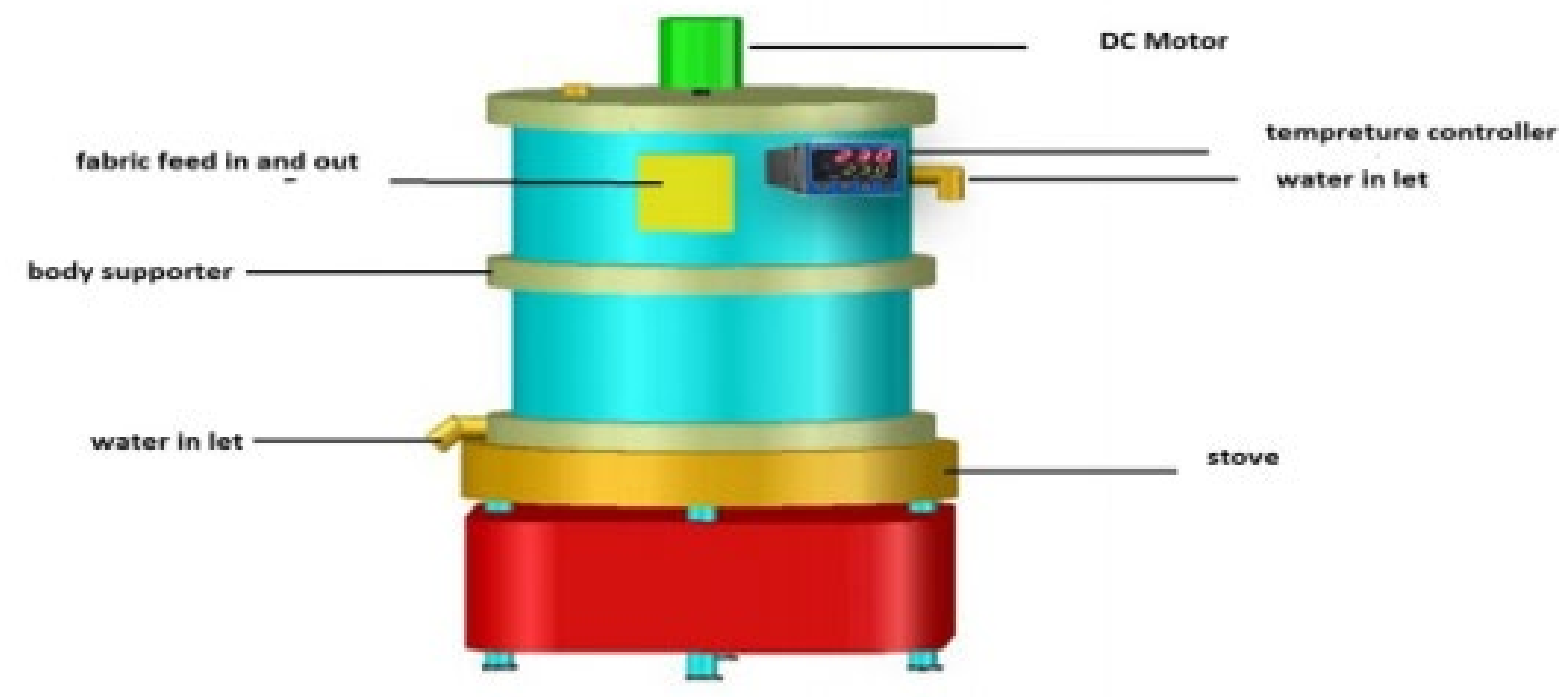

Figure 3: Machine Design. 


\section{Manufacturing Process (Figure 4)}

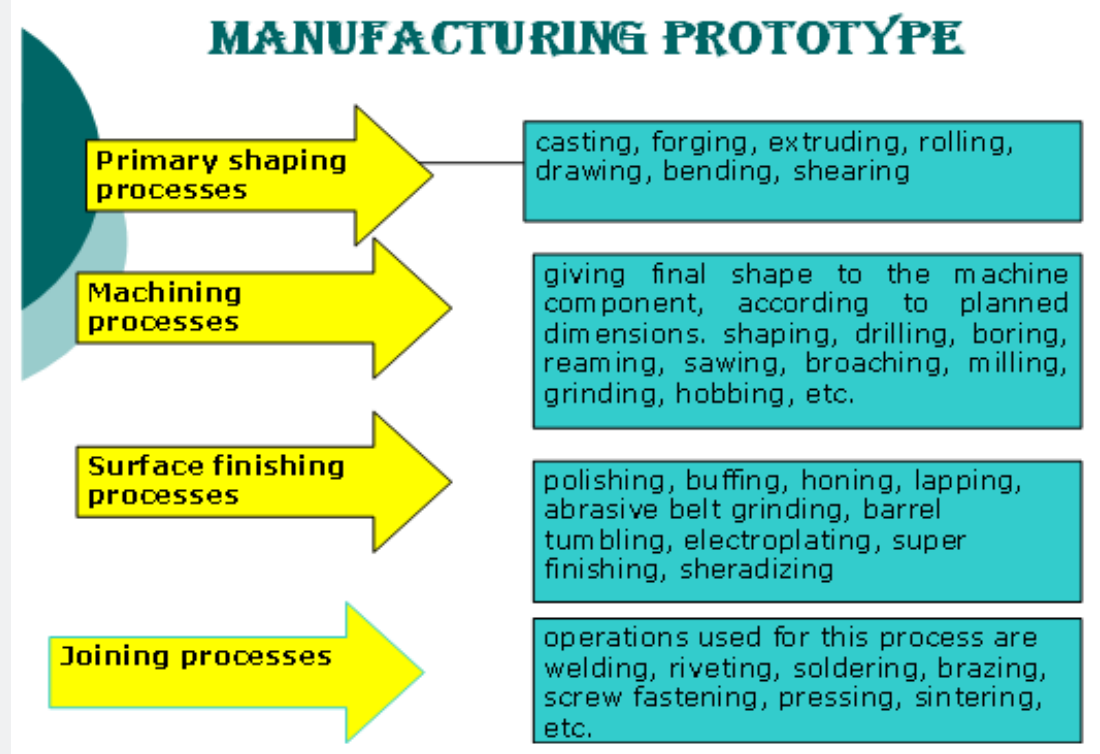

Figure 4: Manufacturing Process.

\section{Working principle of the machine}

In the dyeing and washing process of our design machine their own motion transmission and working principle [14]. In this process add MLR by using the inlet tube up to the required amount of recipe then the stove gives heat to the machine and start the motion from the motor buy using on/off switch and the motor gives to the shaft. The shaft and stirrer are engaged together, so when the shaft rotates the stirrer also rotate [15]. During this time the stirrer stirs simultaneously the dye bath and sample of fabric, therefore the dyeing process are take place uniformly by using the blade of the stirrer and the centrifugal force of the water that is pressurized by the stirrer rotation up to completed the setting time to dyeing process. After this switch off the motor and the stove then eliminate the dyeing waste [16] by opening the derange tube then close the tube when all wastes remove and start the washing process continuously by adding water and detergent by a given MLR ratio and the washing process take place up to remove unfixed dye completely with required temperature and time [17].

\section{Conclusion and Recommendation}

Generally, the present work is devoted to design and manufacturing of laboratory dying machine [18]. Through experimenting different kinds of dying process in laboratory we often use beakers, stirrers, thermometer, stove and so on... for the process [19]. In the process it is recommended to stir well, controlling temperature and washing very well to get the most effective even shade, but in the present condition of EITEX textile dyeing laboratory since this is done manually it is difficult to see the full dying effect. But most importantly the cost to invest in purchasing all this material and the cost to build the washing place is expensive and space is wasted [20-24]. The new design will simplify this problem since it can be built by lower cost and took a small space. It is also can take up to $1 \mathrm{~kg}$ of fabric and it is more durable because its body is made out of low carbon steel. The angle of the stirrer blade is at $45^{\circ}$ so that it can stir effectively and efficiently as a result there will be better evenness on the fabric since the stirring action is continuous until the process ends [25-27]. The machine will also have a digital temperature control system so that the process temperature can be controlled through the whole experiment. The Machine can be done easily, and it is possible to expand or increase its size even to dye a fabric for sale especially it can give and a beneficiary help for small Enterprises workers since it can be done easily without any complication.

\section{References}

1. Arthur D Broadbent (2001) Basic Principles of Textile Coloration. Department de genie chimique, Faculté de génie,Sherbrooke 1-568.

2. Park J (1998) A Practical Introduction to Process Control and Automation in the Batch Dyeing Industry.

3. Hickman WS (1998) Washing Theory and Practice. Rev Prog Coloration 28: 39-60.

4. Duckworth C (1983) Engineering in Textile Coloration. In: Dyers Company Publications Trust, Australia, pp. 574.

5. Schutte MD, Bahr CB, Weetman RJ, Howk RA (1991) Mixing System for Dispersing a compressible Fluid.

6. Weetman RJ (1985) Measurement of fluid forces Mixing apparatus and the control of Mixing Apparatus in Response to Fluid Forces.

7. Weetman RJ, Salzman RN (1981) Impact of side flow on mixing Impeller. 
8. Yahya Faruk (1997) Textile Dyeing and Printing Technology. Madina Printer \& packager, Saudi Arabia.

9. Rahman Moshiur (2008) Wet Processing Technology Part-2.

10. Shennai VA Currently Technical consultant, Former Professor of Textile Chemistry, Department of Chemical Technology, University of Bombay, India.

11. Gao Xiaogang (2005) The Application and Development of Textile Machinery Automatization Technology. Textile Machinery 8(4).

12. Song Jianping (2005) The Technical Innovation and Diversification Strategy of Textile Machinery Manufacturing. Textile Machinery 2(1).

13. Khurmi RS, Gupta JK (2005) Machine Design. New Delhi.

14. Bloch HP, Geitner FK (1983) Machinery Failure Analysis and Troubleshooting. In: $4^{\text {th }}$ (edn)., Gulf Publishing Company, US, pp. 760.

15. Locke JJ (1974) Cobalt Alloy Overlays in a Petro-Chemical Refinery. Cobalt 2: 25-31.

16. Mendenhall MD (1980) Shaft Overlays Proven Effective. pp. 191-192.

17. (1973) Tribology Handbook In: Neale MJ (eds)., $2^{\text {nd }}($ edn)., New York, P. E13.
18. Chrome Plating. sales brochure by Exline, Kansas, US

19. Pyles R (1944) Porous Chromium in Engine Cylinders. Transactions of the ASME, pp. 205-214.

20. Tichvinsky LM, Fischer EG (1939) Boundary Friction in Bearings at Low Loads. Transactions of the ASME 61.

21. Vacca AP (1964) Extended Periods of Overhaul of Diesel Machinery.

22. Mollerus APHJ (1964) Wear Data of Cylinder Liners. study submitted to Ingenieursbureau Lemet Chromium H, Van Der Horst NV.

23. Stinson KW (1996) Diesel Engineering Handbook. In: $12^{\text {th }}($ edn)., Diesel Publications Inc, USA.

24. Eichenour C, Edwards VH (1982) Electromechanical Metalizing Saves Time in Rebuilding Engine Parts. Plant Servicep, p. 49-50.

25. Metal Surface Technology, Addison, US.

26. Treatment of Steel. Republic Steel Corporation Heat, US, P. 22-23.

27. Moffat JD (1975) New Metal Impregnation Technology Solves Friction and Corrosion Problems. NY publications, USA, P. 2-7.

\section{Your next submission with Juniper Publishers will reach you the below assets}

- Quality Editorial service

- Swift Peer Review

- Reprints availability

- E-prints Service

- Manuscript Podcast for convenient understanding

- Global attainment for your research

- Manuscript accessibility in different formats

( Pdf, E-pub, Full Text, Audio)

- Unceasing customer service

Track the below URL for one-step submission https://juniperpublishers.com/online-submission.php 